\title{
Time-varying Channel Estimation for OFDM Systems in the Vehicular Environment
}

\author{
Jiang Peng ${ }^{1}$, Zhinian $\mathrm{Luo}^{1}$ and Bin Liu ${ }^{1}$ \\ 1. College of Computer Science and Electronic Engineering, Hunan University, Changsha, 410082, \\ P.R.China
}

\begin{abstract}
The impacts of vehicular environments on the orthogonal frequency division multiplexing (OFDM) system are investigated, and the effects on channel estimation are studied in this paper. An improved channel estimation scheme is proposed in order to estimate time-varying channels correctly in the vehicular communications such as $802.11 \mathrm{p}$. First time-varying channel is approximated by a generalized complex-exponential basis expansion model (GCE-BEM), and then time-varying channel gain matrix is estimated initially by the training sequence in the preamble and updated iteratively by the data symbols according to the correlation characteristic of the channel response (CR) between the adjacent two data symbols. Basis expansion model (BEM) coefficients are estimated by least square (LS) method. The computer simulations show that the proposed scheme has better bit error rate (BER) and spectrum efficiency performance than the traditional methods.
\end{abstract}

Keywords: OFDM system, time-varying channel estimation, BEM.

\section{Introduction}

OFDM technique has been widely used in modern wireless networks, such as digital video broadcasting- terrestrial/handheld (DVB-T/H), digital audio broadcasting (DAB), wireless local area network (WLAN), worldwide interoperability for microwave access (WiMAX), long term evolution (LTE), for its property of high frequency spectrum efficiency and coping with multipath fading [1]. Moreover, the absence of inter-carrier interference (ICI) allows an easy one-tap equalizer in OFDM systems for linear timeinvariant (LTI) frequency-selective channels [2]. However, when the channel experiences non-negligible time variations resulting from the fast movement of the transmitter or the receiver, each subcarrier in the OFDM symbol undergoes Doppler spreading which will destroy the orthogonality of subcarriers, and as a consequence the ICI is produced [3]-[5].

In high-speed mobile vehicular environment, the fast variation of multipath channels in time domain will lead to Doppler spreading in frequency domain, which is the primary reason for ICI, and the frequency domain channel matrix is not diagonal in that case [6]. The best approach to removing ICI is reducing the symbol length and the sampling period, however, when the symbol length is too short or the sampling period is too small, the overall system may suffer from data inefficiency or bandwidth inefficiency, respectively. Note that the sampling period is the reciprocal of signal bandwidth. Time-varying channel estimation and channel equalization techniques are used widely to mitigate ICI, but the tricky problem of time-vary channel estimation is that the channel coefficients $N \times L$ exceed the maximum observation number $N$, where $N$ is the number of subcarriers of an OFDM symbol and $L$ is the number of multipath. Furthermore, the complexity of channel equalization is very high for OFDM systems in time-varying channel. Linear model and basis extension model (BEM) are widely researched for time-varying channel, because the estimated channel coefficients can be reduced less than the observation number by the use of this model. And least square (LS) or linear minimum mean square error (LMMSE) are utilized normally to estimate the model coefficients [7-8]. However, this methods need more pilots in an OFDM symbol, which decreases the 
spectrum efficiency. In Ref. [9], a novel channel estimation scheme utilizing the data symbols to construct pilots as well as the correlation characteristics between channels within two adjacent symbols is proposed. This scheme owns high spectrum efficiency because of the needless of pilots, and it has better bit error rate (BER) performance than the normal channel estimation methods. In this scheme, however, the equalizer is a one-tap and ICI is not considered.

Based on the aforementioned analysis, an improved and effective time-varying channel estimation scheme for the IEEE 802.11p standard is proposed in this article. In this scheme, time-varying channel is fitted by the generalized complex-exponential basis expansion model (GCE-BEM). Firstly, the second long training sequence in the preamble is used to estimate time-varying channel by LS. Then, the method proposed in Ref. [9] which utilizes the data symbols to construct pilots and the correlation characteristics between channels within two adjacent symbols is used to update time-varying channel gain matrix iteratively. A multi-tap channel equalizer is used to mitigate ICI in the process.

The remainder of this article is organized as follows. In section 2, system models including signal model and BEM model for time-varying channel are described. The proposed scheme of time-varying channel estimation for OFDM systems in the vehicular environment is introduced in section 3 . In section 4, simulation results and analysis are presented. Finally, basic conclusions are drawn in section 5.

\section{System Model}

\subsection{Signal Model}

An OFDM symbol with $N$ subcarriers and $N_{g}$ cyclic prefix (CP) is considered. The transmitted $n$-th time sample of an OFDM symbol is expressed as

$$
x(n)=\frac{1}{\sqrt{N}} \sum_{k=0}^{N-1} \mathrm{X}(k) e^{j 2 \pi k n / N},-N_{g} \leq n \leq N-1,
$$

where $\mathrm{X}(k)$ is the modulated data at the $k$-th subcarrier. Let us denote a $T_{s}$ sampling duration, so that the OFDM symbol duration is then $T_{s y m}=\left(N+N_{g}\right) T_{s}$. For $L$-path time-varying channel, the $n$-th time sample of an OFDM symbol in the receiver after removing $C P$ is expressed as

$$
y(n)=\sum_{l=0}^{L-1} h(n, l) x(n-l)+w(n), 0 \leq n \leq N-1,
$$

where $h(n, l)$ is the $l$-th channel tap in the $n$-th time sample, and $w(n)$ is Gaussian noise.

Let $\boldsymbol{x}=[x(0), \ldots, x(N-1)]^{T}$ and $\boldsymbol{y}=[y(0), \ldots, y(N-1)]^{T}$ be the $m$-th transmitted and received OFDM symbol, respectively, the Eq. 2 can be expressed in matrix-vector form as $\boldsymbol{y}=\boldsymbol{h} \boldsymbol{x}+\boldsymbol{w}$, where $\boldsymbol{w}=[w(0), \ldots, w(N-1)]^{T}$ is a white complex Gaussian noise vector, and $\boldsymbol{h}$ is a $N \times N$ channel gain matrix in time domain with

$$
[\boldsymbol{h}]_{i, j}=\left\{\begin{array}{cc}
h\left(i-1,(i-j+N)_{\bmod N}\right) & (i-j+N)_{\bmod N}<L \\
0 & \text { others }
\end{array} .\right.
$$

The $N \times N$ channel matrix in frequency domain is expressed as $\boldsymbol{H}=\boldsymbol{F} \boldsymbol{h} \boldsymbol{F}^{H}$, where $\boldsymbol{F}$ stands for the $N$ point unitary discrete Fourier transform (DFT) matrix with $[\boldsymbol{F}]_{p, q}=1 / \sqrt{N} e^{-j 2 \pi p q / N}$, and $\boldsymbol{F}^{H}$ is the complex conjugate transpose (Hermitian) matrix of $\boldsymbol{F}$. Thus, OFDM symbol model in frequency domain can be expressed as $\boldsymbol{Y}=\boldsymbol{H} \boldsymbol{X}+\boldsymbol{W}$, where $\boldsymbol{X}=\boldsymbol{F} \boldsymbol{x}, \boldsymbol{Y}=\boldsymbol{F} \boldsymbol{y}$ and $\boldsymbol{W}=\boldsymbol{F} \boldsymbol{w}$.

\subsection{BEM Model for Time-varying Channel}

For time-varying channel, channel matrix in time domain can be approximated by a BEM. Many BEM designs have been proposed to model the time-varying channel, such as discrete Karhuen-Loève BEM (DKL-BEM), complex-exponential BEM (CE-BEM), and polynomial BEM (PBEM) [10-12]. The frequency domain channel matrix for CE-BEM is strictly banded and widely used due to its algebraic simplicity. In this paper, the GCE-BEM that is an optimized model of CE-BEM is used. The basis function of it is expressed as 


$$
[\mathbf{B}]_{n, q}=e^{j 2 \pi \frac{q-Q / 2}{K N} n},
$$

where $K$ is the generalized coefficient, and $Q$ is the order of BEM, which is satisfied $Q>2\left\lceil f_{d} N T_{s}\right\rceil$, where $f_{d}$ is the maximum Doppler shift. $h(n, l)$ in the BEM can be represented as follows [10]:

$$
h(n, l)=\sum_{q=0}^{Q} \mathrm{c}_{q}(l) \mathrm{b}_{q}(n)
$$

where $c_{q}(l)$ is the $(q+1)$-th BEM coefficients of the $l$-th channel tap during one OFDM symbol, and $b_{q}$ is the basis function with size $N \times 1$. Therefore, with the use of BEM, the estimated time-varying channel coefficients can be reduced to $L \times(Q+1)$ while $Q$ is much smaller than $N$, which means that the number of observation data exceeds the estimated data.

As a result, the OFDM symbol model in frequency domain with BEM can be represented as follows [8]:

$$
\boldsymbol{Y}=\mathbf{D} \boldsymbol{X} h^{B}+\boldsymbol{W},
$$

where $\mathbf{D}=\left[D_{0}, \cdots, D_{Q}\right], D_{q}=\boldsymbol{F} \operatorname{diag}\left(b_{q}\right) \boldsymbol{F}^{H}, \boldsymbol{X}=\boldsymbol{I}_{Q+1} \otimes\left(\operatorname{diag}(\boldsymbol{X}) \boldsymbol{F}_{L}\right), h^{B}=\left[c_{0}{ }^{T}, \cdots, c_{Q}{ }^{T}\right]^{T}$. Note that $\otimes$ represents Kronecker product and $\operatorname{diag}(\boldsymbol{X})$ indicates a diagonal matrix with $\boldsymbol{X}$ as its diagonal.

\section{Time-varying Channel Estimation}

In the structure of the IEEE 802.11p standard, each packet consists of preamble including short training symbols and long training symbols, SIGNAL field, and DATA field. The number of subcarriers in one OFDM symbol is 64 , which includes 48 data subcarriers and 4 phase tracking pilot subcarriers. In addition, 11 virtual subcarriers as well as a direct current subcarrier are also added to realize 64-point IFFT.

The second long training sequence is used to estimate the time-varying channel response matrix initially, where it is pseudo noise (PN) symbol after BPSK modulation. Let' $s$ rewrite the Eq. 6 as

$$
\boldsymbol{Y}=\boldsymbol{p} h^{B}+\boldsymbol{d} h^{B}+\boldsymbol{W},
$$

where $\boldsymbol{p}=D_{p} X_{p}$ and $\boldsymbol{d}=D_{d} X_{d}$ represent the corresponding matrix in the subcarriers of pilot and data, respectively. With the use of LS estimator, it is straightforward to obtain the BEM coefficients as

$$
\begin{aligned}
h^{B} & =p^{\dagger} Y_{p} \\
& =h^{B}+p^{\dagger}\left(\boldsymbol{d} h^{B}+W_{p}\right),
\end{aligned}
$$

where $p^{\dagger}$ is the pseudo inverse matrix of $p$ and $Y_{p}$ is the signal of pilots in the receiver. It can be seen through the Eq. 7, when an OFDM symbol is not pilots totally, the pilots will suffer interference from the subcarriers of data. But the ICI is consists of adjacent subcarriers mostly [13-14]. In our scheme, $\boldsymbol{X}$ in Eq. 6 is the second long training sequence, therefore $p^{\dagger} \boldsymbol{d} h^{B}$ is not existed for the absence of data in the training sequence, which means the absence of interference from data to pilots. Time-varying channel gain matrix in time domain and frequency domain can be calculated further by the aforementioned equation.

To update the time-varying channel matrix iteratively, the construct data pilot (CDP) estimator is used. The CDP estimator comprises five steps, i.e., equalization, constructing data pilot, LS estimation, equalization and demapping, and comparison.

The schematic structure of the estimator for time-varying channel is illustrated. In Fig. 1, where $Y_{m}$ and $H_{C D P, m}$ are the $m$-th frequency domain OFDM symbol and corresponding frequency domain channel gain matrix in the receiver, respectively. The detailed explanations of the aforementioned five steps are listed as follows.

Step 1-Equalization. As shown in Fig. 1, CDP estimator iteratively updates the channel estimation employing the previous symbol' s channel response (CR) since the CR of the adjacent two data symbols have high correlation. Therefore, the first step, equalization, is performed as 


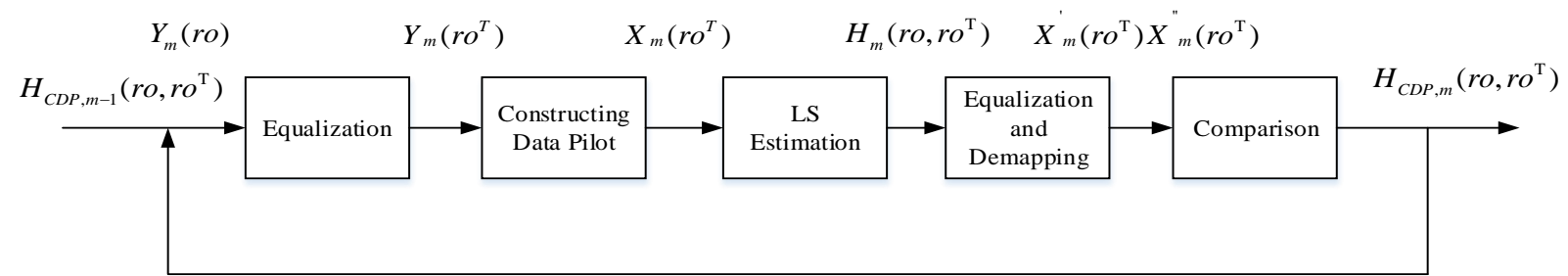

Fig. 1: Schematic structure of the estimator for time-varying channel.

$$
Y_{m}(r o)=\left(H_{C D P, m-1}\left(r o, r o^{T}\right)\right)^{\dagger} Y_{m}(r o),
$$

where $H_{C D P, m-1}\left(r o, r o^{T}\right)$ is the output of the previous estimation process, i.e., $(m-1)$-th symbol' s estimated $\mathrm{CR}$. Note that $r o$ is a column vector representing the index of data subcarriers and the 4 phase tracking pilot subcarriers, and $r o^{T}$ is the transpose of $r o$. As pilots, the data subcarriers will suffer interferences from the 4 phase tracking pilot subcarriers, therefore the column of $H_{C D P, m-1}$ includes the channel information in the 4 phase tracking pilot subcarriers. Then, the ICI caused by the 4 phase tracking pilot subcarriers will be eliminated by equalization. However, the virtual subcarriers and direct current subcarrier are not considered for the generation of ICI.

Step 2-Constructing Data Pilot. After FFT and equalization, we obtain frequency-domain $Y_{m}(r o)$ is then demapped to construct data pilots $X_{m}(r o)$ namely the core of the proposed scheme, in which the 4 phase tracking pilot is used with value 1 directly. The principle of the CDP estimator is depicted in Fig. 2. Owing to the impacts of noise and other interferences, $Y_{m}$ is possibly located into the wrong regions. Therefore, $X_{m}$ is easily to be demapped to the incorrect constellation points. With the assist of demapping, the impact from noise and interferences may be partially alleviated. The remaining error can be further mitigated in the following steps by exploiting the correlation characteristics between channels within two adjacent symbols.

Step 3-LS Estimation. The constructed data pilot $X_{m}$ is then employed to calculate the $m$-th data symbol' s BEM coefficients by using Eq. 8, i.e., the LS estimation, where $D\left(r o, r o^{T}\right)_{p}$ is used in Eq. 6. Note that the 4 phase tracking pilots are also used for time-varying channel estimation. And the frequency domain gain matrix can be obtained by the aforementioned equation.

Step 4-Equalization and Demapping. In the following two steps, the high correlation characteristic is exploited again. $H_{m}$ is first used to equalize $Y_{m-1}$ such that

$$
Y_{m-1}^{\prime}(r o)=\left(H_{m}\left(r o, r o^{T}\right)\right)^{\dagger} Y_{m-1}(r o) \text {. }
$$

$Y_{m-1}$ is then equalized by $H_{C D P, m-1}$, i.e., the previous symbol' s estimated CR. The equalized $Y_{m-1}^{\prime \prime}(r o)$ is given by

$$
Y_{m-1}^{\prime \prime}(r o)=\left(H_{C D P, m-1}\left(r o, r o^{T}\right)\right)^{\dagger} Y_{m-1}(r o) \text {. }
$$

To compare $Y_{m-1}$ and $Y_{m-1}^{\prime \prime}$ they should be demapped to the corresponding constellation points $X_{m-1}($ ro $)$ and $X_{m-1}^{\prime \prime}(r o)$ according to the type of modulation.

Step 5-Comparison. As aforementioned, we have discussed that the two adjacent data symbols have high correlation. Hence, if $X_{m-1}(k) \neq X_{m-1}^{\prime \prime}(k)$, it indicates that the k-th subcarrier's $X_{m}(k)$, which demapped after Eq. 10 is incorrect and we should define that $H_{C D P, m}\left(k, r o^{T}\right)=H_{C D P, m-1}\left(k, r o^{T}\right)$, i.e., the previous symbol' s estimated CR. Otherwise, if $X_{m-1}^{\prime}(k)=X_{m-1}^{\prime \prime}(k)$, we have $H_{C D P, m}\left(k, r o^{T}\right)=H_{m}\left(k, r o^{T}\right)$.

It is noticeable that the condition for the previous channel estimation steps is $m>1$ and $m=1$ is excluded. The reason is that the two long training symbols are binary phase shift keying (BPSK) modulated to 1 and -1 . However, data symbols are possibly modulated by other schemes instead of BPSK. The solution of this problem is introduced in Ref [9], there is no longer to do more. But, considering the ICI in the second long training sequence, the order of channel gain matrix in the Eq. 10 and 11 should be changed to $N \times N$ when $m=1$. 


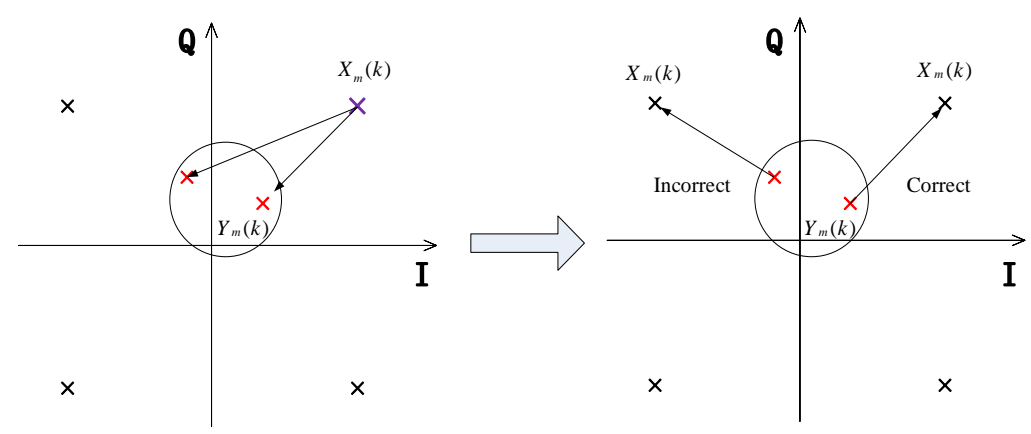

Fig. 2: Principle of the CDP estimator.

\section{Simulation Results and Analysis}

In this section, bit error ratio (BER) simulation of the proposed time-varying channel estimation algorithm is conducted. To compare with our proposed scheme, the LS and initial CDP schemes in Ref. [9] are taken as two representative examples.

In order to simulate the vehicle environment, channel models of three representative scenarios proposed in Ref. [15-16] are adopted in this paper. In Table 1, the channel parameters of the three scenarios, VTV Expressway Oncoming, VTV Urban Canyon Oncoming, RTV Urban Canyon, are listed, noting that the data vector represents the corresponding scenario as before, where n/a means not-applicable. The maximum Doppler shift of the three scenarios is set as $1000 \mathrm{~Hz}, 500 \mathrm{~Hz}$ and $300 \mathrm{~Hz}$, respectively. And the corresponding velocity of the vehicle is $105 \mathrm{~km} / \mathrm{h}, 48 \mathrm{~km} / \mathrm{h}$ and $48 \mathrm{~km} / \mathrm{h}$, respectively. The modulation of every path is Rayleigh distribution. According to Table 1, we set the corresponding GCE-BEM parameters as $Q=2, K=2$, and $L=5$. And the packet structure of the IEEE 802.11p standards is applied in the simulation. The coefficients about OFDM symbol are set as $N=64, N_{g}=16, T_{s}=0.1$ us.

Figs. 3-5 show the comparison of the LS, initial CDP, and modified CDP schemes in BER performance of 4QAM under three vehicular environments, i.e., VTV Expressway Oncoming, VTV Urban Canyon Oncoming, and RTV Urban Canyon, respectively. To evaluate the performance of the IEEE 802.11p with different packet lengths, the numbers of OFDM symbols are chosen to be 100 and 200. The figures depict that smaller packet length is beneficial for the BER performance, especially for CDP schemes. This is because that with the packet length increasing the correlation of channel between data symbols will decrease. As expected, the LS scheme keeps on a relatively high level of BER, while much smaller BERs are achieved by CDP schemes. And, obviously, the modified CDP scheme owns better BER performance to the initial CDP scheme. The primary reason of this is that the ICI cancellation is considered in the modified CDP scheme.

According to the simulation results, with higher Doppler shift, the V2V channels exhibit stronger timevarying characteristic and owns higher BER level. As for the RTV Urban Canyon scenario, which has the difference of BER performance in different packet length is obvious than lower Doppler shift. This is because that when the Doppler shift is higher, the channel correlation characteristics between adjacent OFDM data will drop. Besides, according to the simulation parameters, because of the needless of pilots, the spectrum efficiency of modified CDP channel estimation is higher than the data-aided channel estimation methods.

Table 1: Channel Parameters of the Three Scenarios

\begin{tabular}{cll}
\hline Tap No. & \multicolumn{1}{c}{ Tap Power $(\mathrm{dB})$} & Delay Value $(u \mathrm{~s})$ \\
\hline 1 & {$[0,0,0]$} & {$[0,0,0]$} \\
2 & {$[-6.3,-10.0,-11.5]$} & {$[0.1,0.1,0.1]$} \\
3 & {$[-25.1,-17.8,-19.0]$} & {$[0.2,0.2,0.2]$} \\
4 & {$[-22.7,-21.1,-25.6]$} & {$[0.3,0.3,0.3]$} \\
5 & {$[\mathrm{n} / \mathrm{a},-26.3,-28.1]$} & {$[\mathrm{n} / \mathrm{a}, 0.4,0.5]$} \\
\hline
\end{tabular}




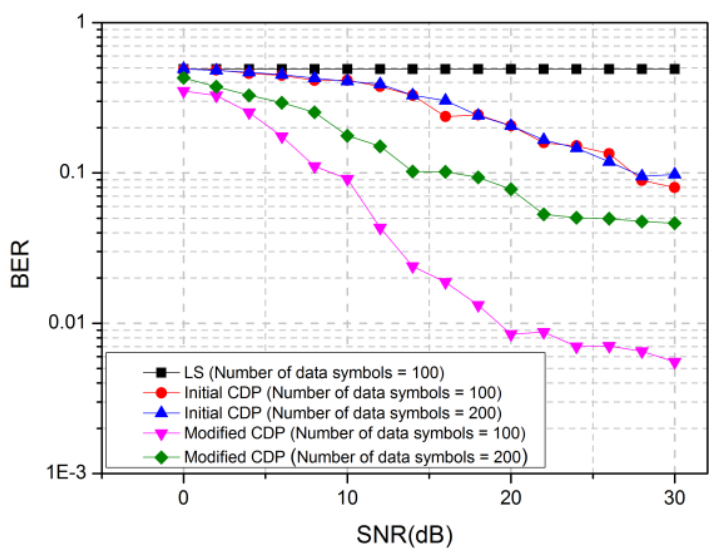

Fig. 3: Comparison of the BER performance of the LS, initial CDP and modified CDP schemes in 4QAM modulation (VTV Expressway Oncoming).

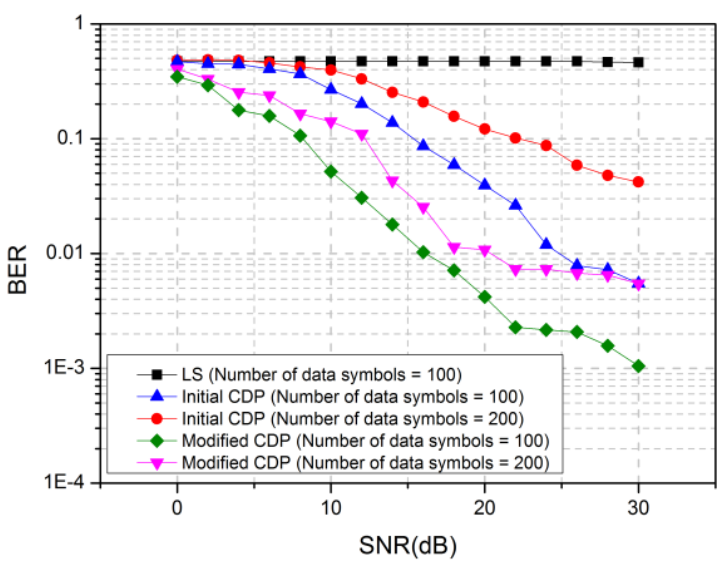

Fig. 4: Comparison of the BER performance of the LS, initial CDP and modified CDP schemes in 4QAM modulation (VTV Urban Canyon Oncoming).

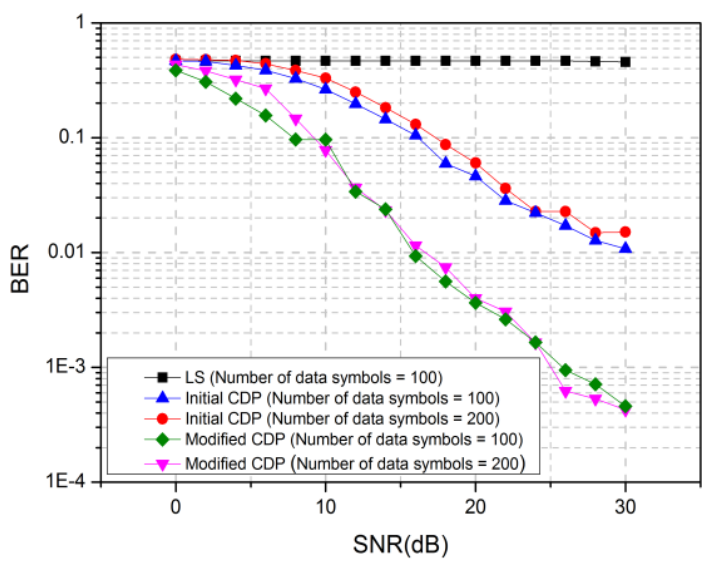

Fig. 5: Comparison of the BER performance of the LS, initial CDP and modified CDP schemes in 4QAM modulation (RTV Urban Canyon). 


\section{Conclusion}

A modified estimation scheme for time-varying channels applied to the IEEE 802.11p standard is proposed. Firstly, time-varying channel is approximated by the GCE-BEM. Then the time-varying channel coefficients are estimated by the LS algorithms with the training sequence in the preamble initially and updated by the CDP iteratively. In order to take the high-speed mobile environment into account, we used the corresponding channel models introduced by Ref. [15]-[16]. Simulation results show that the proposed time-varying channel estimation scheme owes higher BER performance and spectrum efficiency. Furthermore, the CDP estimator may also be applied to tracking CFO, and the joint CFO tracking and timevarying channel estimation are obtained. For the high-speed mobile environment, the CFO in packets may be different for the varying of Doppler shift. In addition, the BER performance will be better by channel coding and interleaving techniques. For the next step study, the proposed channel estimation method used for vehicular environments may be improved in complexity and efficiency by equalization and iteration, and the application for other channel environments and wireless communication standards is also consider.

\section{Acknowledgements}

This work is supported by the National Nature Science Foundation of China No. 61371115.

\section{References}

[1] A. Stamoulis, S. Diggavi, and N. Al-Dhahir, “Intercarrier interference in MIMO OFDM,” IEEE Trans. Signal Process., vol. 50, no. 10, pp. 2451- 2464, Oct. 2002.

[2] Sari, H., Karam, G., \& Jeanclaude, I. (1995). Transmission techniques for digital terrestrial TV broadcasting. IEEE Communications Magazine, 33(2), 100- 109.

[3] T. Pollet, M. Van Bladel, and M. Moeneclaey, " BER sensitivity of OFDM systems to carrier frequency offset and Wiener phase noise,” IEEE Trans. Commun., vol. 43, no.2/3/4, pp. 191-193, Feb./March/April 1995.

[4] H. Steendam and M. Moeneclaey, "Sensitivity of Orthogonal Frequency-Division Multiplexed Systems to Carrier and Clock Synchronisation Errors," Signal Processing, vol. 80, pp. 1217-1229, 2000.

[5] Robertson, P., \& Kaiser, S. (1999, September). The effects of Doppler spreads in OFDM (A) mobile radio systems.In Proceedings of the IEEE VTC-99 Fall: (pp. 329- 333), Amsterdam, The Netherlands.

[6] Zijian Tang; Leus, G., "Pilot schemes for time-varying channel estimation in OFDM systems," in Signal Processing Advances in Wireless Communications, 2007. SPAWC 2007. IEEE 8th Workshop on, vol., no., pp.1-5, 17-20 June 2007

[7] Kyungchul Kwak; Sungeun Lee; Hyunkee Min; Sooyong Choi; Daesik Hong, "New OFDM Channel Estimation with Dual-ICI Cancellation in Highly Mobile Channel," inWireless Communications, IEEE Transactions on, vol.9, no.10, pp.3155-3165, October 2010

[8] Zijian Tang; Cannizzaro, R.C.; Leus, G.; Banelli, P., "Pilot-Assisted Time-Varying Channel Estimation for OFDM Systems," in Signal Processing, IEEE Transactions on , vol.55, no.5, pp.2226-2238, May 2007

[9] Zijun Zhao, Xiang Cheng, Miaowen Wen, et al., "Channel Estimation Schemes for IEEE 802.11p Standard," Intelligent Transportation Systems Magazine, IEEE, vol.5, no.4, pp.38-49, winter 2013

[10] K.A.D. Teo, S. Ohno, Optimal MMSE finite parameter model for doubly-selective channels, In: Proc. of Global Telecommunications Conference, 2005 (GLOBECOM’ 05), IEEE, St. Louis, MO, Dec. 2, 2005: 3503-3507.

[11] H.A. Cirpan, M.K. Tsatsanis, Maximum likelihood blind channel estimation in the presence of Doppler shifts, Signal Processing, 1999, 47(6): 1559-1569. 
[12] S. Tomasin, A. Gorokhov, H.B. Yang, et al., Iterative interference cancellation and channel estimation for mobile OFDM, Wireless Communications, 2005, 4(1): 238-245.

[13] Li, Xin; Fan, Pingzhi, Modified clustered comb pilot-aided fast time-varying channel estimation for OFDM system, Journal of Modern Transportation, 2095-087X 2012

[14] Yuping Zhao; Haggman, S.-G., Intercarrier interference self-cancellation scheme for OFDM mobile communication systems, in Communications, IEEE Transactions on, vol. 49, no. 7, pp.1185-1191, Jul 2001,

[15] G. Acosta-Marum and M. A. Ingram, “ Six time-and frequency-selective empirical channel models for vehicular wireless LANs,” IEEE Veh. Tech. Mag., vol. 2, no. 4, pp. 4- 11, Dec. 2007.

[16] G. Acosta-Marum, “ Measurement, Modeling, and OFDM synchronization for the wideband mobile-to-mobile channel,” Ph.D. dissertation, Georgia Inst. Technol., Atlanta, GA, 2007. 